\title{
Localized surface-plasmon polaritons in disordered nanostructured metal surfaces: Shape versus Anderson-localized resonances
}

\author{
José A. Sánchez-Gil* \\ Instituto de Estructura de la Materia, Consejo Superior de Investigaciones Científicas, Serrano 121, 28006 Madrid, Spain
}

(Received 12 June 2003; published 29 September 2003)

\begin{abstract}
The electromagnetic wave scattering from disordered nanostructured metal surfaces supporting surfaceplasmon polaritons (SPP) is studied by means of fully retarded scattering formulations. We investigate the two physical mechanisms which may underlie the excitation of localized SPP: Anderson localization and shape resonances. The former mechanism is discarded since plane-wave excited, localized SPP are observed in the absence of proper Anderson localization of SPP. In contrast, a detailed analysis of the near field for various ensembles of surface realizations permits us to identify SPP shape resonances typically occurring at sub100-nm grooves or ridges, the latter being significantly stronger.
\end{abstract}

DOI: 10.1103/PhysRevB.68.113410 PACS number(s): 73.20.Mf, 42.25. $-\mathrm{p}$, 68.37.Uv, 78.68. $+\mathrm{m}$

Surface-plasmon polaritons (SPP) have attracted a great deal of attention in recent years. These are evanescent electromagnetic (EM) waves bounded to metal-dielectric interfaces due to oscillations of the electron plasma in the metal. ${ }^{1}$ A very rich phenomenology is exhibited when SPP are excited on nanostructured metals: particularly, SPP confinement may take place on either periodic ${ }^{2}$ or disordered ${ }^{3-5}$ systems. The latter phenomenon relates to the observation of localized optical modes, not only on metal surfaces supporting SPP, ${ }^{3}$ but also on different nanosystems supporting particle plasmons such as colloidal aggregates. ${ }^{6,7}$ Such localized optical modes are manifested by large and highly localized EM fields, which lie on the basis of the EM enhancement in surface-enhanced Raman scattering (SERS) and other nonlinear, surface optical processes, ${ }^{8}$ and in turn in SERS singlemolecule detection. ${ }^{9,10}$

In the case of nanoparticle aggregates, it has been theoretically argued that localized optical modes stem from Anderson localization of particle plasmons. ${ }^{7,11}$ Nonetheless, to our knowledge there is no clear theoretical evidence as to what is the physical mechanism leading to localized optical modes in disordered nanostructured metals supporting SPP, wherein the effects of retardation and, in turn, of radiative leakage are crucial. There are two obvious candidates: shape SPP resonances; or configuration resonances (low-probability, high-transmission modes for given frequency/realization ${ }^{12,13}$ ) associated with the Anderson localization of SPP propagating along the disordered surface.

In this Brief Report, we investigate the physical mechanism underlying the roughness-induced excitation of localized SPP (LSPP) on disordered nanostructured metal surfaces. We restrict our attention to one-dimensional (1D) surfaces without loss of generality, as will become evident below, because of computational constraints on the numerical calculations needed to deal with the rigorous theoretical formulation involved. First, we study under either plane wave (PW) or SPP illumination (of frequency $\omega$ ) the scattering from a randomly rough metal surface $z=\zeta(\mathbf{r})=\zeta(x)$ [Fig. 1(a)]. We exploit the impedance boundary condition ${ }^{14}$ on a planar surface in order to obtain the reduced Rayleigh equation for the scattering amplitude of the only nonzero component of the magnetic field in $p$ polarization (along the $y$ axis) in vacuum $(z>\zeta)$ :

$$
\begin{aligned}
H^{>}(x, z)= & H_{i}^{>}(x, z)+\int_{-\infty}^{\infty} \frac{d q}{2 \pi} R(q, \omega) \\
& \times \exp \left[i q x+i \alpha_{o}(q, \omega) z\right],
\end{aligned}
$$

where $\alpha_{o}(q, \omega)=\left[\left(\omega^{2} / c^{2}\right)-q^{2}\right]^{1 / 2}$ for $|q| \leqslant \omega / c$ and $\alpha_{o}(q, \omega)=i\left[q^{2}-\left(\omega^{2} / c^{2}\right)\right]^{1 / 2}$ for $|q|>\omega / c . \quad H_{i}^{>}(x, z)$ $=\exp \left[i(\omega / c)\left(x \sin \theta_{0}-z \cos \theta_{0}\right)\right]$ in the case of PW illumination with angle of incidence $\theta_{0}$; or $H_{i}^{>}(x, z)=\exp [i k(\omega) x$ $\left.-\beta_{0}(\omega) z\right]$ for a SPP impinging from the negative $x$ axis, with wave-vector components $k(\omega)=(\omega / c)\left[1-\epsilon^{-1}\right]^{1 / 2}$ and $\beta_{0}(\omega)=-\imath \alpha_{o}[k(\omega)]=(\omega / c)[-\epsilon]^{-1 / 2}, \epsilon=\epsilon(\omega)$ being the frequency-dependent dielectric function of bulk silver ${ }^{15}$ (neglecting dissipative losses). The resulting $k$-space (KS) integral equation is

$$
\begin{aligned}
R(p, \omega)= & G_{0}(p, \omega) V\left(p \mid p_{i}\right) \\
& +G_{0}(p, \omega) \int_{-\infty}^{\infty} \frac{d q}{2 \pi} V(p \mid q) R(q, \omega),
\end{aligned}
$$

where $p_{i} \equiv(\omega / c) \sin \theta_{0} \quad(\mathrm{PW})$ or $k(\omega) \quad(\mathrm{SPP}), G_{0}(p, \omega)$ $\equiv i \epsilon\left[\epsilon \alpha_{0}(p, \omega)+i(\omega / c)(-\epsilon)^{1 / 2}\right]^{-1}$ is the Green's function of the SPP on the unperturbed surface, and the scattering potential $V(p \mid q) \equiv \beta_{0}(\omega) \hat{s}(p-q)$ has been introduced that is proportional to the Fourier transform of the surface impedance $s(x)$, which can in turn be linearly connected with the actual surface roughness $\zeta(x) .{ }^{14}$ The details of the formulation are given elsewhere. ${ }^{16}$

In Fig. 1(b) we show the surface electric-field intensity for both PW (normal incidence) and SPP illumination at the wavelength $\lambda=2 \pi c / \omega=620 \mathrm{~nm}$, for a Ag surface profile extracted from an ensemble of realizations obeying Gaussian statistics and Gaussian correlation (GC) function with correlation length $a=102.8 \mathrm{~nm}$ and $\delta=51.4 \mathrm{~nm}$ [Fig. 1(a)]. These parameters ensure the presence of surface features in the sub-100-nm range, which is known to favor the PW excitation of SPP: ${ }^{4,5}$ indeed, LSPP are found with large values of $|E(x, \zeta(x))|^{2}\left[>10^{2}\right.$ times the electric-field intensity of the incident PW in Fig. 1(b) at $x \approx-2.9 \mu \mathrm{m}]$. In contrast, $|E(x, \zeta(x))|^{2}$ in Fig. 1(b) for an incident SPP shows no clear evidence of LSPP (nearly exponentially) decaying along the 


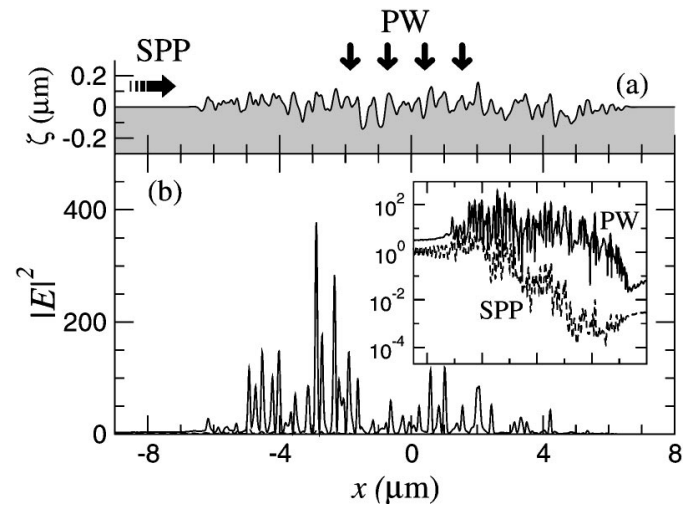

FIG. 1. (a) Illustration of the scattering geometry (incident PW or SPP). Surface profile extracted from an ensemble of GC realizations with $a=102.8 \mathrm{~nm}, \delta=51.4 \mathrm{~nm}$, and $L=12.6 \mu \mathrm{m}$. (b) Surface electric-field intensities calculated (KS) for the surface profile depicted in (a) and $\lambda=620 \mathrm{~nm}$. Inset: semilog scale. Solid curve: incident PW; dashed curves (barely visible in the linear scale): incident SPP.

SPP propagating direction. This reveals that LSPP can be excited on a rough metal surface, whereas launching a SPP along exactly the same disordered metal surface does not lead to a resonant excitation related to Anderson localization. However, to rule out this physical mechanism, further evidence should be obtained.

For this purpose we calculate: (a) the frequency dependence of the SPP transmission coefficient $T(\omega)$ for a given realization; (b) the length dependence of the ensemble average of the SPP transmission coefficient (actually $\langle\ln T\rangle$ ), the SPP reflection coefficient $\langle R\rangle$, and the total integrated radiated power $\langle S\rangle$ (resulting from SPP coupling into outgoing propagating waves in vacuum). $T, R$, and $S$ are normalized by the power carried by the incident SPP, ${ }^{16}$ so that energy conservation imposes $R+T+S=1$. The results are presented in Fig. 2. First, it is evident from the structureless, fairly flat behavior of $T(\omega)$ for one realization [circles in Fig. 2(a)] that no resonant frequencies are found within a wide frequency range. Conversely, we have also calculated $T$ for different realizations at fixed frequency (not shown here), and no SPP resonances are observed either. As an example, $T(\omega)$ for the same frequency interval (normalized to the center frequency) is shown for single-mode propagation along a 1D surface-disordered waveguide in the regime of Anderson localization ${ }^{17}$ (the rough waveguide parameters are chosen so that $\langle\ln T\rangle$ is comparable to that for our SPP transmission problem); large- $T$ peaks linked to resonances are found.

We turn next to the analysis of the $L$ dependence of the ensemble-averaged quantities: $\langle\ln T\rangle,\langle R\rangle$, and $\langle S\rangle$ are shown in Figs. 2(b) and 2(c). Note that $\langle\ln T\rangle$ decays monotonically. Nonetheless, this decay does not correspond to a substantial increase in the SPP reflection coefficient $\langle R\rangle$, as it would be the case in real 1D Anderson localization [for which $S \equiv 0$ and $R+T=1$; see the solid curves in Figs. 2(b) and 2(c)]. It is due just to radiative leakage, $\langle S\rangle$, the predominant SPP attenuation mechanism, rather than to coherent SPP-SPP (back)scattering, as can be seen in Fig. 2(c), thus precluding the onset of Anderson localization. There-

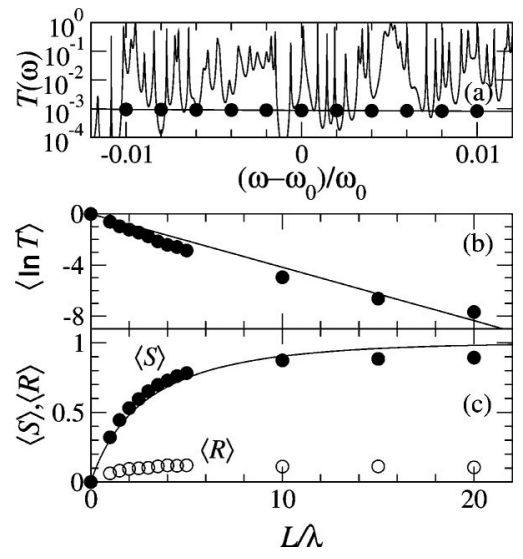

FIG. 2. KS calculations for SPP incidence. (a) SPP transmission coefficient as a function of renormalized frequency for a single realization as in Fig. 1(a) (circles), but with length $L=6.3 \mu \mathrm{m}$. Length dependence for fixed $\lambda=620 \mathrm{~nm}$ of (b) $\langle\ln T\rangle$ (circles) and (c) $\langle R\rangle$ (hollow circles) and $\langle S\rangle$ (filled circles) obtained by averaging over an ensemble of $\left(N_{p}=200\right) \mathrm{GC}$ realizations [with the same roughness parameters as in Fig. 1(a)]. The results for single-mode transmission through a surface-disordered waveguide are also included for comparison (solid curves), except for the meaningless $\langle S\rangle \equiv 0$ in (c).

fore, LSPP appear on random nanostructured metal surfaces for which there is no Anderson localization of SPP. ${ }^{18}$ The predominance of radiative leakage has been confirmed by our numerical calculations for different roughness parameters and also for self-affine fractals with nanoscale lower cutoff: ${ }^{4}$ in all cases for a wide frequency range. In particular, if the surface roughness strength is weakened, either by lowering $\delta$ down to the nanometer scale or by using a larger incident wavelength $\lambda=2 \mu \mathrm{m}$, both attenuation mechanisms diminish, but with fairly large ratio $\langle S\rangle /\langle R\rangle \gg 1$.

What is the physical mechanism underlying the roughness-induced excitation of LSPP? Let us thoroughly characterize (PW-excited) LSPP on nanostructured metal surfaces, in connection with topography. To this end, it is more convenient to employ the exact scattering formulation of the real-space (RS), surface integral equations resulting from the application of Green's second integral theorem, for the scattering geometry in $p$ polarization being studied [see Fig. 1(a) for PW excitation]. The magnetic-field amplitude in vacuum can be written as

$$
\begin{aligned}
H^{>}(\mathbf{r})= & H_{i}^{>}(\mathbf{r})+\frac{1}{4 \pi} \int_{-\infty}^{\infty} d x^{\prime}\left[\gamma^{\prime} H\left(x^{\prime}\right) \frac{\partial G_{o}\left(\mathbf{r}, \mathbf{r}^{\prime}\right)}{\partial n^{\prime}}\right. \\
& \left.-L\left(x^{\prime}\right) G_{o}\left(\mathbf{r}, \mathbf{r}^{\prime}\right)\right], \quad z>\zeta(x),
\end{aligned}
$$

where $G_{o}$ is the two-dimensional, real-space Green's function, and the normal derivative is defined as $\partial / \partial n \equiv(\hat{\mathbf{n}} \cdot \boldsymbol{\nabla})$, with $\hat{\mathbf{n}} \equiv \gamma^{-1}(-\partial \zeta / \partial x, 0,1)$ and $\gamma=\left[1+(\partial \zeta / \partial x)^{2}\right]^{1 / 2}$. In Eq. (3), the surface magnetic field $H(x)$ and its normal derivative $\gamma L(x)$ play the role of source functions (numerically obtained as detailed in Ref. 4) from which all the EM field 


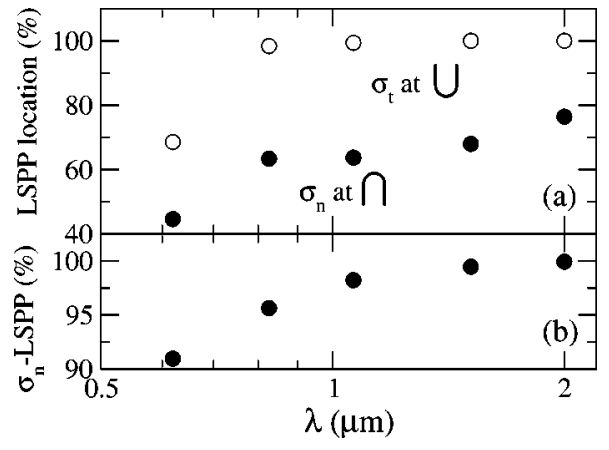

FIG. 3. (a) Percentage of LSPP located at surface maxima $\left(\sigma_{n}\right.$, filled circles) and minima ( $\sigma_{t}$, hollow circles), along the $\lambda=0.5$ $-2 \mu \mathrm{m}$ range, obtained from RS calculations of the surface electric-field components for GC surface realizations with $a$ $=25.7 \mathrm{~nm}, \quad \delta=128 \mathrm{~nm}$, and $L=5.14 \mu \mathrm{m}$. (b) Percentage of $\sigma_{n}$-type LSPP, obtained as in (a).

components both in vacuum and in the metal are calculated (dissipative losses are now fully accounted for through the complex value of the $\mathrm{Ag}$ dielectric function ${ }^{15}$ ). Monte Carlo simulation calculations have been carried out for the intensity of the normal and tangential electric-field components $\sigma_{n, t}(x)=\left|E_{n, t}(x, \zeta(x))\right|^{2} /\left|\mathbf{E}^{(i)}(x, \zeta(x))\right|^{2}$ for ensembles of $\mathrm{GC}$ and self-affine fractal surface realizations with different roughness parameters, all of them presenting sub-100-nm features known to favor LSPP excitation. ${ }^{4,5}$ It should be emphasized that our results for $\sigma(x)$ yield statistics ${ }^{19}$ in qualitative and quantitative agreement with the experimental results from photon scanning tunneling microscopy (PSTM) images. ${ }^{20}$ We look for the percentage of LSPP (with given electric polarization and intensity enhancements above a threshold $\sigma_{c}=10^{2}$ ) that is connected to surface (nano)ridges and (nano)grooves.

The results for a typical ensemble of GC surfaces are shown in Fig. 3. The surface electric field of LSPP is predominantly polarized along the normal component $(>90 \%)$. But what is most striking is the correlation with the surface features in Fig. 3(a): $\sim 60 \%$ of such normal LSPP appear at surface maxima, the rest being at other locations (not surface minima). Conversely, the tangential LSPP (only $<10 \%$ of the total) concentrate at surface minima $(\sim 90 \%)$. All these features are preserved throughout the spectral region being studied, the percentage of tangentially polarized LSPP tending to vanish in the near-IR $(\sim 2 \%$ at $\lambda>1064 \mathrm{~nm})$, as expected from the metal boundary conditions.

Thus the statistical analysis of $\sigma(x)$ indicates that LSPP exist predominantly at surface maxima and minima. But what do these LSPP look like? We have examined the nearfield patterns of LSPP for many different ensembles (GC surfaces and also self-affine fractals), finding striking similarities in strong correlation with their location at either grooves or ridges. In Figs. 4(a) and 4(c), the near-electricfield $(\log )$ intensity map of a LSPP in the vicinity of a surface groove is plotted with normal incidence and $\lambda$ $=620 \mathrm{~nm}$; recall that the total illuminated surface is much longer $(L=5.14 \mu \mathrm{m})$ than shown. The near-field pattern suggests that a SPP is trapped near the groove bottom with a
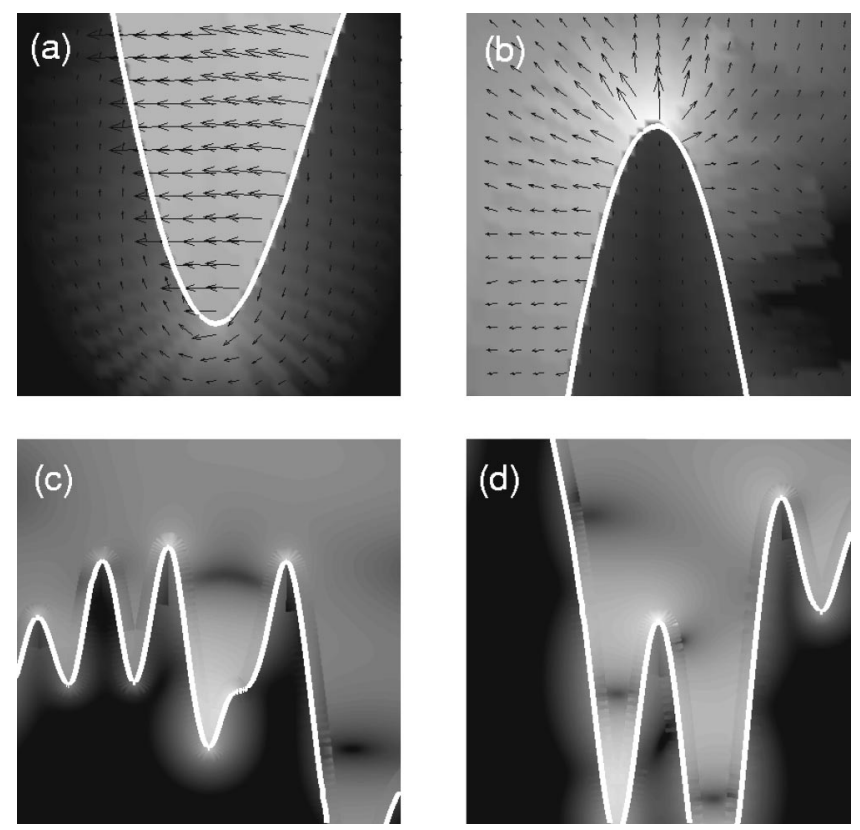

FIG. 4. Near-field images (RS calculations) of the enhancement of the $p$-polarized electric-field intensity $\left(\theta_{0}=0^{\circ}\right)$ in a log scale close to a GC surface realization (with roughness parameters as used in Fig. 3) in an area $0.5 \times 0.5 \mu \mathrm{m}^{2}$, where a LSPP is observed at either (c) a groove or (d) a ridge, zoomed in $\left[60 \times 60 \mathrm{~nm}^{2}\right]$ in (a) and (b), respectively. The surface profile is superimposed as a white curve (and extends over $L=5.14 \mu \mathrm{m}$ ). Grey scales span from (black) $\log \sigma=-2$ in (a) and (b), or from $\log \sigma=0$ in (c) and (d), to 3 (white).

dipolelike [see near-electric-field polarization zoomed in Fig. 4(a)], opposite charge concentration on either groove wall. Such an electric-field distribution responds to a favorable configuration allowed by the continuity conditions, charge oscillations inside the metal, and the corresponding normalelectric polarization of the SPP. And despite the fact that $E_{n}^{S P P} / E_{t}^{S P P} \propto|\epsilon+1|^{1 / 2}$, the configuration leads to a large value of $\sigma_{t}$ at the surface minimum, ${ }^{1}$ which in turn constitutes the fingerprint of tangential LSPP in the previous statistical analysis, together with the two maxima of $\sigma_{n}$ symmetrically located at both sides of the groove and contributing to nearly $40 \%$ of the uncorrelated $\sigma_{n}$ LSPP [Fig. $3(\mathrm{a})$. In light of the predominantly normal polarization as shown in Fig. 4(a), it is more appropriate to call them groove-LSPP. These groove-LSPP are similar to LSPP found in periodic arrays of metallic semicylinders. ${ }^{2}$

The near-electric-field (log-intensity and polarization) map corresponding to a LSPP at a surface ridge is shown in Figs. 4(b) and 4(d). The electric field is fairly symmetric and normal to the surface, with a large peak at the very tip of the surface [as expected from the statistical analysis of $\sigma_{n}$ LSPP in Fig. 3(a)], resembling a monopolar configuration: this configuration is also enforced by the boundary conditions, SPP polarization, and non-forbidden charge distribution at the very end of the metal tip at adjacent walls. Note that the minimum spatial width of (both ridge and groove) LSPP along the surface profile is $\sim \lambda_{S P P} / 2\left[\lambda_{S P P} \equiv \lambda(\epsilon\right.$ 

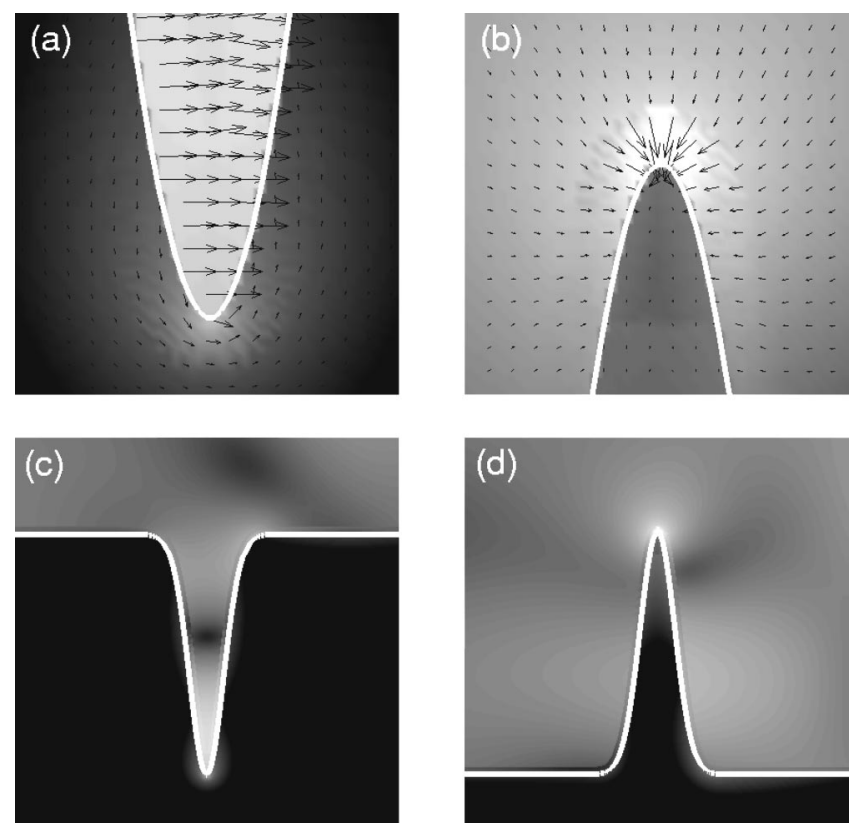

FIG. 5. Same as in Fig. 4, but for Gaussian defects of 1/e halfwidth $A=25.7 \mathrm{~nm}$ and height $h= \pm 257 \mathrm{~nm}\left(\theta_{0}=40^{\circ}\right)$. All grey scales span from $\log \sigma=-1$ (black) to 2 (white).

$+1)^{1 / 2} / \epsilon^{1 / 2}$ being the planar SPP wavelength $\left.{ }^{1}\right]$, compatible with the experimental PSTM images., ${ }^{3,20}$

Further evidence in support of SPP shape resonances is given in Fig. 5, where the near-electric-field map is shown for isolated, metal Gaussian defects with dimensions close to those of the typical grooves and ridges in the random GC surfaces being studied. The electric-field distribution near the surface protuberance (indentation) exhibits the same mo- nopolar (dipolar) LSPP configuration observed above for ridges (grooves) in the randomly rough surface profiles (Fig. 4). Alternatively, we have also verified that the latter LSPP are preserved when the rough surface profiles are flattened at regions beyond a certain lateral distance $d$ from the surface (nano)ridge/groove where a LSPP appears. The robustness of LSPP is thus another argument in favor of shape SPP resonances, and against Anderson-localized SPP resonances, which are more sensitive to the entire surface profile. ${ }^{13} \mathrm{Fi}-$ nally, we have analyzed the role of absorption either by artificially increasing the imaginary part of the dielectric function or by lowering $\omega$ below the onset of interband transitions (for $\mathrm{Au}$ and $\mathrm{Cu}$ ), leading to similar LSPP with smaller EM field enhancements.

In summary, we have investigated through full electromagnetic calculations the excitation of LSPP in disordered nanostructured metal surfaces, with the aim of determining the underlying physical mechanism: Anderson localization of SPP or SPP shape resonances. Our results rule out the former mechanism, whereas unequivocally connecting LSPP with SPP shape resonances occurring at either grooves or ridges. The conclusions have been verified for a wide spectral range in the visible and near-IR, and should be applicable to any disordered nanostructured metal configuration supporting SPP. This is not the case of subwavelength nanoparticle aggregates, where localized optical excitations are observed and interpreted in the quasistatic approach as localized surface plasmons. ${ }^{7,11}$

This work was supported in part by the Spanish DGIMCyT (Grants Nos. BFM2000-0806 and BFM2001-2265), and by NSF Grant No. INT-0084423. The author is grateful to J. V. García-Ramos, E. R. Méndez, A. A. Maradudin, and M. Nieto-Vesperinas for fruitful discussions.
*Electronic address: j.sanchez@iem.cfmac.csic.es

${ }^{1}$ H. Raether, Surface Polaritons on Smooth and Rough Surfaces and on Gratings (Springer-Verlag, Berlin, 1988).

${ }^{2}$ F.J. García-Vidal and J.B. Pendry, Phys. Rev. Lett. 77, 1163 (1996).

${ }^{3}$ S.I. Bozhevolnyi, I.I. Smolyaninov, and A.V. Zayats, Phys. Rev. B 51, 17916 (1995); S.I. Bozhevolnyi and V. Coello, ibid. 58, 10 899 (1998).

${ }^{4}$ J.A. Sánchez-Gil, J.V. García-Ramos, and E.R. Méndez, Phys. Rev. B 62, 10515 (2000).

${ }^{5}$ J.A. Sánchez-Gil, J.V. García-Ramos, and E.R. Méndez, Opt. Express 10, 879 (2002).

${ }^{6}$ S.I. Bozhevolnyi, V.A. Markel, V. Coello, W. Kim, and V.M. Shalaev, Phys. Rev. B 58, 11441 (1998).

${ }^{7}$ S. Grésillon, L. Aigouy, A.C. Boccara, J.C. Rivoal, X. Quelin, C. Desmaret, P. Gadenne, V.A. Shubin, A.K. Sarychev, and V.M. Shalaev, Phys. Rev. Lett. 82, 4520 (1999).

${ }^{8}$ V.M. Shalaev, Phys. Rep. 272, 61 (1996); A.K. Sarychev and V.M. Shalaev, ibid. 335, 275 (2000).

${ }^{9}$ S. Nie and S.R. Emory, Science 275, 1102 (1997).

${ }^{10}$ K. Kneipp, Y. Wang, H. Kneipp, L.T. Perlman, I. Itzkan, R.R. Dasari, and M.S. Feld, Phys. Rev. Lett. 78, 1667 (1997).

${ }^{11}$ M.I. Stockman, S.V. Faleev, and D.J. Bergman, Phys. Rev. Lett.
87, 167401 (2001).

${ }^{12}$ I. M. Lifshits, S. A. Gredeskul, and L. A. Pastur, Introduction to the Theory of Disordered Systems (Wiley, New York, 1988), Chap. 7.

${ }^{13}$ J. A. Sánchez-Gil and V. Freilikher, Phys. Rev. B 68, 075103 (2003).

${ }^{14}$ The connection between surface electric and magnetic fields at rough vacuum/metal interfaces through a surface impedance eliminates the need for explicitly dealing with the EM field in the metal. See, e.g., A. A. Maradudin, in Topics in Condensed Matter Physics, edited by M. P. Das (Nova, New York, 1994).

${ }^{15}$ Handbook of Optical Constants of Solids, edited by E. D. Palik (Academic, Orlando, 1985).

${ }^{16}$ J.A. Sánchez-Gil and A.A. Maradudin, Phys. Rev. B 60, 8359 (1999).

${ }^{17}$ J.A. Sánchez-Gil, V. Freilikher, I. Yurkevich, and A.A. Maradudin, Phys. Rev. Lett. 80, 948 (1998).

${ }^{18}$ This in turn justifies our reducing the dimensionality of the model in the numerical calculations, for it is well known that this favors Anderson localization.

${ }^{19}$ J.A. Sánchez-Gil and J.V. García-Ramos, Chem. Phys. Lett. 367, 361 (2003).

${ }^{20}$ S.I. Bozhevolnyi and V. Coello, Phys. Rev. B 64, 115414 (2001). 